\title{
Flexoelectric Fracture-Ratchet Effect in Ferroelectrics
}

\author{
Kumara Cordero-Edwards, ${ }^{1,5,}{ }^{*}$ Hoda Kianirad, ${ }^{2}$ Carlota Canalias, ${ }^{2}$ Jordi Sort, ${ }^{3,4}$ and Gustau Catalan ${ }^{1,4, \dagger}$ \\ ${ }^{1}$ Catalan Institute of Nanoscience and Nanotechnology (ICN2), \\ CSIC and BIST, Campus UAB, Bellaterra, 08193 Barcelona, Catalonia \\ ${ }^{2}$ Department of Applied Physics, KTH-Royal Institute of Technology, Roslagstullsbacken 21, 10691 Stockholm, Sweden \\ ${ }^{3}$ Departament de Física, Universitat Autònoma de Barcelona (UAB), Edifici Cc, E-08193 Bellaterra, Spain \\ ${ }^{4}$ Institució Catalana de Recerca i Estudis Avançats (ICREA), Pg. Lluís Companys 23, E-08010 Barcelona, Catalonia \\ ${ }^{5}$ DPMC-MaNEP, Université de Genéve, Quai Ernest-Ansermet 24, 1211 Geneva, Switzerland
}

(Received 4 October 2018; revised manuscript received 20 February 2019; published 2 April 2019)

\begin{abstract}
The propagation front of a crack generates large strain gradients and it is therefore a strong source of gradient-induced polarization (flexoelectricity). Herein, we demonstrate that, in piezoelectric materials, a consequence of flexoelectricity is that crack propagation is helped or hindered depending on whether it is parallel or antiparallel to the piezoelectric polar axis. The discovery of crack propagation asymmetry proves that fracture physics cannot be assumed to be symmetric in polar materials, and indicates that flexoelectricity should be incorporated in any realistic model.
\end{abstract}

DOI: 10.1103/PhysRevLett.122.135502

Crack propagation causes materials to break, and forms the basis of fracture physics, a vital element of materials science and engineering as it determines the mechanical resilience of devices [1,2]. Fascinatingly, controlled cracking has also been proposed as a mechanism for device nanopatterning [3], turning the harnessing of crack propagation into a constructive pursuit. In the specific case of piezoelectric and ferroelectric materials, fracture physics is additionally important because voltage-induced strains cause the appearance and propagation of microcracks that result in material fatigue and the ultimate failure of piezoelectric transducers $[4,5]$. The fracture physics of piezoelectrics is therefore a fundamental problem with important practical ramifications. Here we show that crack-generated flexoelectricity causes in ferroelectrics an original valvelike or "crack filter" behavior, whereby crack propagation is facilitated or impaired depending on the sign of the ferroelectric polarization. In other words, the toughness of ferroelectrics is, like their polarization, switchable.

Flexoelectricity (coupling between strain gradient and polarization) [6-8] has disruptive consequences for the mechanical physics of materials, enabling new behaviors [9-12]. For example, it has recently been predicted [12] and demonstrated [13] that ferroelectrics can have an asymmetric mechanical response to inhomogeneous deformations. Because fracture fronts concentrate the biggest local deformations that a solid can withstand, flexoelectricity is also expected to affect fracture behavior. For example, the flexoelectric fields generated by cracks are strong enough to be able to trigger the self-repair process in bone fractures [14]. The present work demonstrates that, due to the interplay between flexoelectricity and ferroelectricity crack propagation in ferroelectrics is asymmetric and switchable, so that cracks propagating parallel to the ferroelectric polarization become longer than those traveling against it.

In the present experiment, Vickers indentation tests were performed on a $\mathrm{Rb}$-doped $\mathrm{KTiOPO}_{4}$ (RKTP) single crystal with the polarization in plane. We chose this ferroelectric because it is uniaxial, and thus ferroelastic effects can be excluded. RKTP is also a technologically relevant material, commonly used as a frequency conversion device in nonlinear optics [15,16]. For such applications, a bulk periodic domain pattern with alternating domain orientations (periodic poling) is created in the crystal. The procedure for this is well established [15], which facilitates the in-plane poling of the crystal. Poling of antiparallel domains on the same crystal was used in order to ensure that geometrical effects such as a slight tilt or miscut of the crystal surface did not affect the results. By poling two domains of antiparallel orientation, indents could be performed on domains of opposite polarity on the same crystal surface and in the same experiment, as sketched in Fig. 1(a). That way, the effect of alternating polarity was tested without affectation from any other spurious effect such as variations in sample geometry or chemistry.

Mechanical tests were conducted by applying sets of 200 and $300 \mathrm{mN}$ loads, with the orientation of the indenter being such that two of its four corners were parallel to the polar axis and the other two perpendicular. In order to control for statistical fluctuations in fracture toughness, 30 indents for each force (15 for each domain polarity) were performed, with each indent generating four cracks along the parallel, antiparallel, and perpendicular directions. In total, 240 cracks were hence analyzed. The radial crack lengths, from the corners of the indents [see inset 

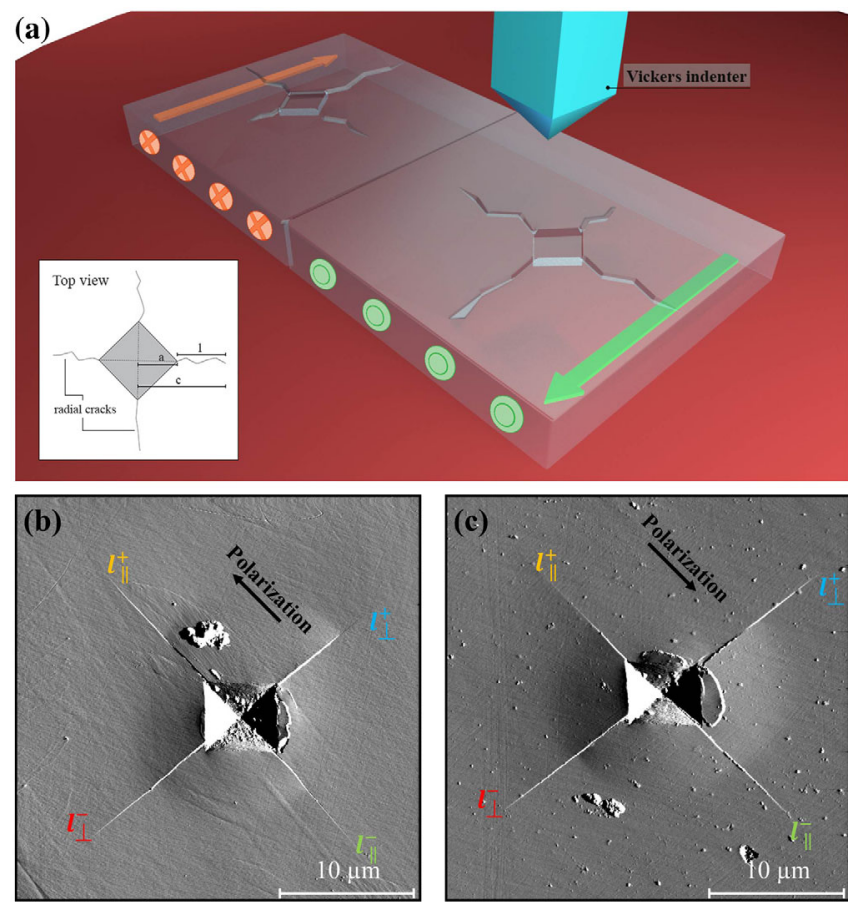

FIG. 1. (a) Schematic of the Vickers indentation test showing the top view of typical radial crack propagation for indentation fracture toughness measurement with corresponding crack $(l)$ and diagonal lengths $(2 a)$. AFM topography of Vickers indent in RKTP showing the radial crack propagation for (b) up and (c) down polarization.

in Fig. 1(a)], were measured with an optical microscope and atomic force microscopy (AFM) immediately after indentation. A sketch of the experiment is in Fig. 1(a), and two indentation samples can be seen in Figs. 1(b) and 1(c).
After measuring the length of the cracks $(l)$, the length asymmetry along the polar axis was calculated for each indentation. To verify that the results were not artifacts, we also measured the asymmetry in the direction perpendicular to the polar direction, where in theory there should be none. We define the asymmetry coefficient as

$$
\% \text { Asy }=\frac{l^{+}-l^{-}}{\langle l\rangle} 100,
$$

where $l^{+}$is the crack length parallel to the polarization, and $l^{-}$is the crack length antiparallel to the polarization (up or down in the plan-view photos). For cracks perpendicular to the poling direction, + and - designate right or the left directions, respectively, in the plan-view photos. The average crack length is $\langle l\rangle \equiv\left[\left(l^{+}+l^{-}\right) / 2\right]$. Positive (negative) asymmetry indicates a longer (shorter) crack than the average. When cracks have the same length, the asymmetry coefficient is zero.

Figure 2(a) shows the asymmetry of the cracks perpendicular to the polar axis. For these, as expected, there is no asymmetry within statistical error. This lack of perpendicular asymmetry provides a safety check for the robustness of the experimental results. In contrast to the perpendicular cracks, Fig. 2(b) shows that cracks parallel to the poling direction are asymmetric: for $P^{+}$domains, a positive asymmetry is measured, and the asymmetry is reversed for the $P^{-}$domains. In other words: crack length parallel to the polarization is always greater than crack length antiparallel to the polarization, irrespective of the polarity of the domain.
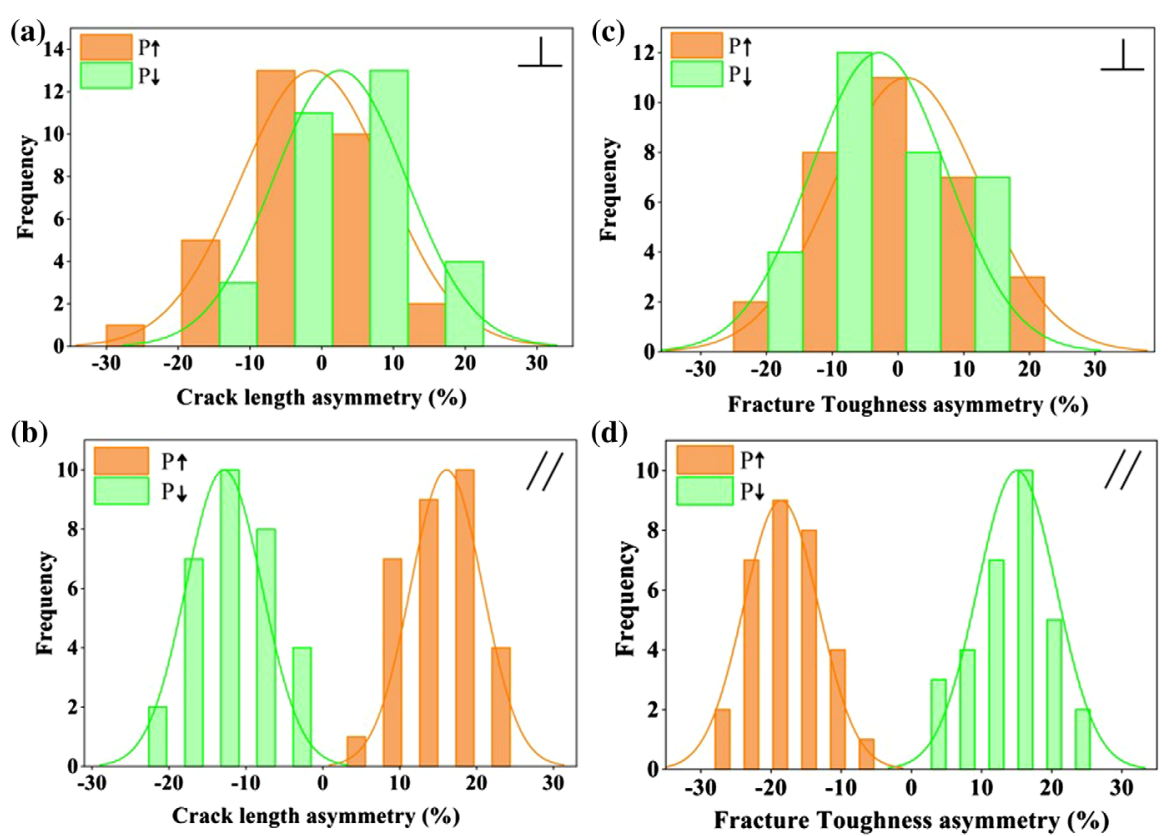

FIG. 2. Crack length asymmetry (a) perpendicular and (b) parallel to the polar axis. Fracture toughness asymmetry (c) perpendicular and (d) parallel to the polar axis. 
The asymmetry of crack length can be used to quantify the asymmetry in fracture toughness, the stress intensity required for creating a crack [17]. Fracture toughness is given by [18]

$$
K_{\mathrm{IC}}=0.016\left(\frac{E}{H}\right)^{\frac{1}{2}}\left(\frac{F}{c^{\frac{3}{2}}}\right), \quad H=\frac{F}{2 a^{2}},
$$

where $E$ is the Young modulus, $H$ the Vickers hardness, $F$ the indent load, $c$ is the distance from the center of the indentation impression to the tip of the crack, and $2 a$ is the diagonal of the indent [see inset in Fig. 1(a)]. Using the values obtained from our tests, $K_{\text {IC }}$ was obtained for each crack, and using the expression (1) the asymmetries were calculated.

Figures 2(c) and 2(d) show the asymmetry for the perpendicular and parallel direction, respectively. As explained, there is asymmetry only along the polar axis, i.e., when ferroelectric and flexoelectric polarizations are parallel (crack propagating in the same direction as the ferroelectric polarization), or antiparallel (crack propagating in the opposite direction as the ferroelectric polarization). The average value of the fracture toughness for cracks parallel to the polarization was $\sim 0.24 \pm 0.02 \mathrm{MPam}^{\frac{1}{2}}$, whereas for the ones antiparallel to the polarization it was $\sim 0.29 \pm 0.03 \mathrm{MPa} \mathrm{m}^{\frac{1}{2}}$. In other words, in ferroelectric RKTP, fracture toughness is enhanced (yielding to shorter cracks) by $20 \%$ when flexoelectricity and ferroelectricity are antiparallel compared to when they are parallel.

As discussed earlier, since all indentations are performed under the exact same geometrical conditions (same surface, same indenter, same experiment), the asymmetry cannot be a geometrical artifact. The fact that the crack-length asymmetry is reversed for domains of opposite polarization implies that the origin is linked to polarity. Differences in surface adsorbates or near-surface defects can be excluded; even if such differences did exist (and none should be expected given that the polarization is in plane), each pair of cracks is generated in the same spot and encounters identical surface conditions. The asymmetry in crack propagation is therefore intrinsic and linked to polarity: ferroelectricity acts as a sort of fracture "valve" that can be switched to facilitate or impair crack propagation.

The basis of the asymmetry is the interplay between flexoelectricity and piezoelectricity [11-13]. The local deformation at the tip of the crack generates a flexoelectric polarization that may be parallel or antiparallel to the ferroelectric polarization, resulting in different mechanical response [12]. For ferroelectrics, there is in theory an additional consideration, which is that the flexoelectric field near the tip of the crack may be large enough to cause local switching of the polarization $[19,20]$, thus providing an additional path for energy dissipation that further reduces the available energy for mechanical fracture.
This process, akin to transformation toughening, is known as switching-induced toughening [21-23]. Switchinginduced toughening has so far been studied in ferroelastic-ferroelectrics (i.e., ferroelectric materials where mechanical stress can switch the direction of the polar axis), but flexoelectricity in principle also enables purely ferroelectric $\left(180^{\circ}\right)$ switching in nonferroelastic uniaxial ferroelectrics [19]. Here we examine the extent to which such effect can contribute to the observed cracking asymmetry of our samples.

Considering a uniaxial ferroelectric, and adding a flexoelectric term to the energy balance, switching should occur when

$$
f_{i j k l} \epsilon_{j, k l} \Delta P_{i}+E_{i} \Delta P_{i} \geq 2 P_{s} E_{c},
$$

where $f_{i j k l}$ is the flexocoupling tensor, $\in_{j, k l}$ is the strain gradient, and $\Delta P_{i}$ are the changes in the spontaneous polarization during the switching, $P_{s}$ is the magnitude of the spontaneous polarization, and $E_{c}$ the coercive electric field. Since there is no external electric field, we can discard the second term, and $\Delta P_{i}=2 P_{s}$ for $180^{\circ}$ domain switching [22]. The condition for switching thus simplifies to $f_{i j k l} \in_{j, k l} \geq E_{c}$. In other words, switching happens when the flexoelectric field (left side of the equation) exceeds the coercive field (right-side term).

To estimate the size of the switched region, we have considered only the longitudinal, transverse, and shear components of the strain gradient, assuming flexocoupling coefficients of the order of $f=10 \mathrm{~V}$, as generally observed for ceramics $[8,24]$. With these simplifications, and for a crack propagating along the polar axis, $x_{3}$, switching should occur in the region of the ferroelectric crystal that satisfies the condition

$$
\left(\frac{\partial \in_{33}}{\partial x_{3}}+\frac{\partial \in_{11}}{\partial x_{3}}+\frac{\partial \in_{31}}{\partial x_{1}}\right) \geq \frac{E_{c}}{f} .
$$

Considering the coercive field of RKTP $\left(E_{c}=3.7 \times 10^{6} \mathrm{~V} \mathrm{~m}^{-1}\right)$ [25], and with the aforementioned simplifications, a total strain gradient of $\sim 3.7 \times 10^{5} \mathrm{~m}^{-1}$ is theoretically required to induce switching in RKTP. To see whether such strain gradients are reached in the vicinity of the crack, we have used elastic theory to calculate the strain field [26]

$$
\varepsilon_{i j}^{e l}=\frac{1+v}{E} \sigma_{i j}-3 \frac{v}{E} \sigma_{m} \delta_{i j},
$$

where $\sigma_{i j}$ is the stress applied to the crack in each direction, and its expression depends on the propagation modes; $\sigma_{m}$ is the average stress; $E$ is the Young's modulus; and $\nu$ is the Poisson ratio. Focusing on crack mode I (tensile loading), the stress fields in this type of crack are given by the following equations, 

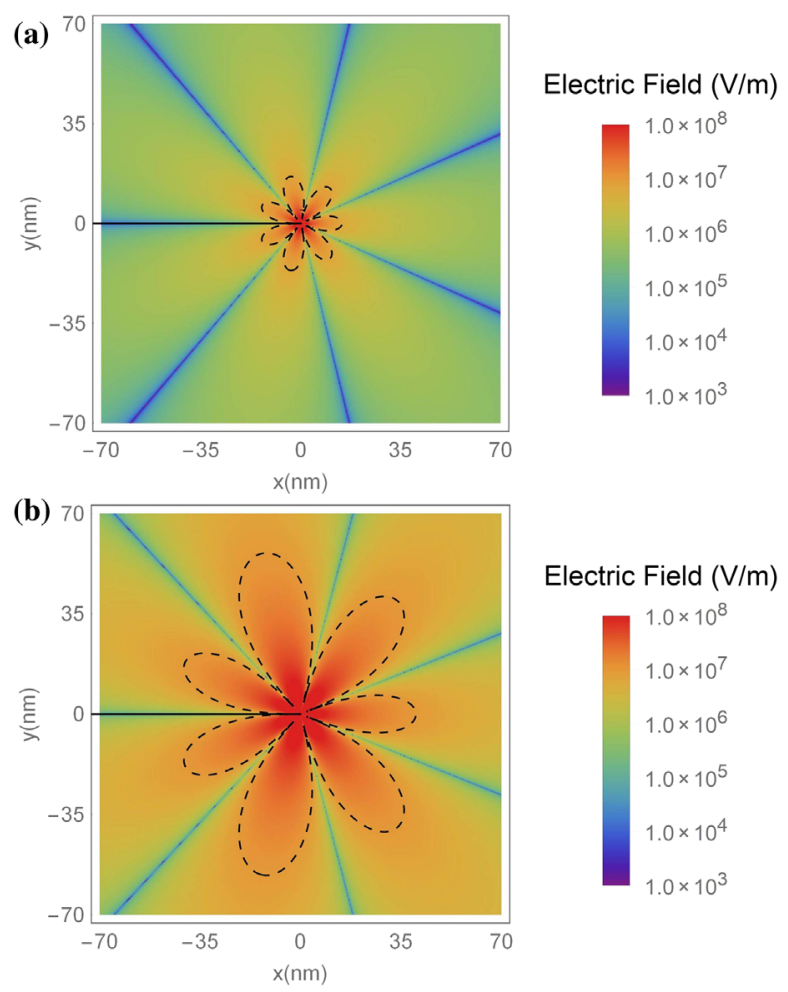

FIG. 3. Calculated distribution of the flexoelectric field around the apex of a crack in (a) RKTP, and (b) in LN. The black line marks the region where the gradient-induced electric field is strong enough to be able to induce local switching of the polarization.

$$
\begin{gathered}
\sigma_{11}=\frac{K_{I}}{\sqrt{2 \pi r}} \cos \frac{\theta}{2}\left(1-\sin \frac{\theta}{2} \sin \frac{3 \theta}{2}\right), \\
\sigma_{22}=\frac{K_{I}}{\sqrt{2 \pi r}} \cos \frac{\theta}{2}\left(1+\sin \frac{\theta}{2} \sin \frac{3 \theta}{2}\right), \\
\tau_{12}=\frac{K_{I}}{\sqrt{2 \pi r}} \cos \frac{\theta}{2} \sin \frac{\theta}{2} \cos \frac{3 \theta}{2},
\end{gathered}
$$

where $K_{I}$ is the intensity factor (fracture toughness for this calculation). Transforming Eqs. (6)-(8) to Cartesian coordinates and using Mathematica [27] for the calculations, we have computed analytically the strain field in Eq. (5) (more details in the Supplemental Materials [28]), and the strain gradient associated with it. The value used for the intensity factor (fracture toughness) was the one obtained in this study, $K_{I}=0.29 \mathrm{MPa} \mathrm{m}^{1 / 2}$; all other values were taken from the literature and are listed in Table 1.
The calculated flexoelectric field map around a crack tip in RKTP is plotted in Fig. 3(a). The dashed line outlines the region within which flexoelectricity is large enough to induce local switching of the polarization. The calculated size of this switching region $(\sim 20 \mathrm{~nm})$, however, is rather small. It is at the edge of thermodynamic stability of a switched domain embedded in a nonswitched matrix [20,34,35], so switchback is almost certain to happen, plus the domain size is also close to the resolution limit of piezoresponse force microscopy (PFM). We examined the cracks by PFM finding no evidence of $180^{\circ}$ local switching near them.

In order to look for evidence of crack-induced flexoelectric switching, we turn to another uniaxial ferroelectric, lithium niobate (LN) with a bigger flexoelectric coefficient (see Table 1). Using Eqs. (5)-(8), and the coefficients in Table 1, we mapped the flexoelectric field [Fig. 3(b)] and found that the theoretical switching radius is $\sim 55 \mathrm{~nm}$ around the tip, which is bigger than that of KTP. This prediction was experimentally tested in a crystal of $\mathrm{LN}$, $y$ cut, indented in the same conditions as with the RKTP sample.

The lateral piezoresponse force microscopy (LPFM) images of the resulting indent and cracks are shown in Fig. 4. In LN, the easy fracture plane is at $60^{\circ}$ with respect to the polar axis, and the cracks tend to zigzag instead of following a clean straight line along the polar axis. Although this makes it impossible to reliably measure and compare their lengths, it does not affect their ability to generate flexoelectric fields. Indeed, the PFM images in Figs. 4(b) and 4(c) show that cracks with a propagation component antiparallel to the polarization induce local $180^{\circ}$ switching, leaving a trail of needle domains in the crack's wake. This "flexoelectric switching" is analogous to the mechanical writing of ferroelectric domains using AFM tip indentation [19]. The mechanical consequences are profound: since switching dissipates energy, the cracks that switch polarization dissipate more energy and thus cannot grow as long as those that do not. Fracture patterns in ferroelectrics must therefore be asymmetric.

In summary, the interaction between flexoelectricity and ferroelectricity in fracture fronts leads to qualitatively new phenomena. First, crack propagation can switch ferroelectric polarity. Second, and conversely, ferroelectric polarity affects crack propagation, making it asymmetric. These findings may have practical implications, as they suggest that fatigue due to microcracking could be mitigated or enhanced according to the poling direction of the ferroelectric. Crack-diode-like functionality also offers a new

TABLE I. Elastic, dielectric and flexoelectric coefficients used in our calculations for the 2D maps.

\begin{tabular}{lccccccc}
\hline \hline Material & $\mathrm{E}_{11}(\mathrm{Gpa})$ & $\mathrm{E}_{33}(\mathrm{Gpa})$ & $\mathrm{E}_{31}(\mathrm{Gpa})$ & $\varepsilon_{r}$ & $f(\mathrm{~V})$ & $\mu=\varepsilon_{r} \varepsilon_{0} f(\mathrm{nC} / \mathrm{m})$ & $E_{c}(\mathrm{KV} / \mathrm{cm})$ \\
\hline LN & $277[29]$ & $231[29]$ & $80[29]$ & $30[30]$ & $40[13]$ & 11 & $210[31]$ \\
RKTP & $165[32]$ & $188[32]$ & $42[32]$ & $13[33]$ & 10 & 1.2 & $37[25]$ \\
\hline \hline
\end{tabular}




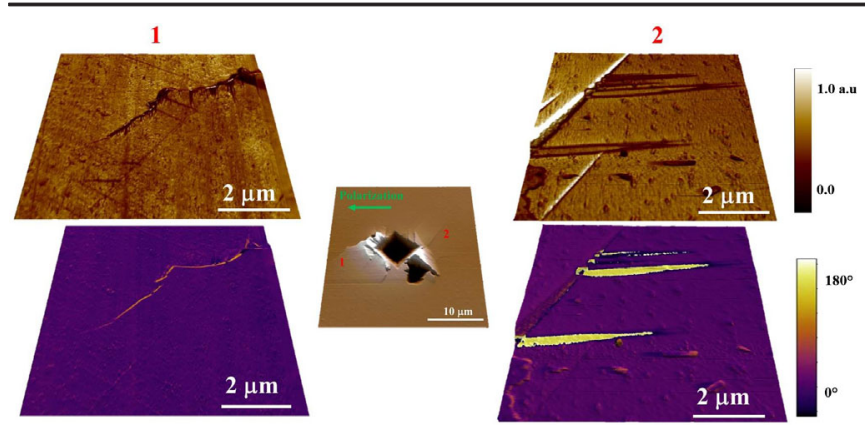

FIG. 4. (Center) AFM topography of Vickers indent in LN $y$ cut showing the radial crack propagation. LPFM amplitude and phase of crack propagating parallel (left) and antiparallel (right) showing local switching as the crack propagates opposite to the polarization of the crystal.

degree of freedom for crack-based nanopatterning [3]. From a fundamental point of view, the discovery implies that the assumption of mechanical inversion symmetry is fundamentally wrong for situations involving inhomogeneous deformation of piezoelectric materials. The results demonstrate that flexoelectricity has to be taken into account in any realistic model of fracture in such materials.

The authors thank Dr. Neus Domingo for valuable discussions. K. C.-E. and G.C. acknowledge ERC Starting Grant No. 308023, MINECO Grant No. MAT2016-77100-C2-1-P from Spain and AGAUR Grant No. 2017-SGR-00579 from Catalonia. ICN2 is supported by the Severo Ochoa program from Spanish MINECO (Grant No. SEV-2017-0706), and also funded by the CERCA Programme / Generalitat de Catalunya. H. K. and C. C. acknowledge the Swedish Research Council for generous support. The work of J.S. has been partially funded by the 2017-SGR-292 project from the Generalitat de Catalunya and the MAT2017-86357-C3-1-R project from the Spanish Ministerio de Economía y Competitividad (MINECO), cofinanced by the 'Fondo Europeo de Desarrollo Regional (FEDER)'.

*Corresponding author.

rohini.corderoeduards@unige.ch

${ }^{\dagger}$ Corresponding author. gustau.catalan@icn2.cat

[1] D. Broek, Elementary Engineering Fracture Mechanics, 4th ed. (Martinus Nijhoff Publishers, Netherlands, 1986).

[2] D. Francois, A. Pineau, and A. Zaoui, Mechanical Behaviour of Materials, 2nd ed. (Springer Press, New York, 2012).

[3] K. H. Nam, I. H. Park, and S. H. Ko, Patterning by controlled cracking, Nature (London) 485, 221 (2012).

[4] S. Kim and Q. Jiang, Microcracking and electric fatigue of polycrystalline ferroelectric ceramics, Smart Mater. Struct. 5, 321 (1996).
[5] J. Shieh, J. E. Huber, and N. A. Fleck, Fatigue crack growth in ferroelectrics under electrical loading, J. Eur. Ceram. Soc. 26, 95 (2006).

[6] S. Kogan, Piezoelectric effect during inhomogeneous deformation and acoustic scattering of carriers in crystals, Sov. Phys. Solid State 5, 2069 (1964).

[7] E. Cross, Flexoelectric effects: Charge separation in insulating solids subjected to elastic strain gradients, J. Mater. Sci. 41, 53 (2006).

[8] P. Zubko, G. Catalan, and A. K. Tagantsev, Flexoelectric effect in solids, Annu. Rev. Mater. Res. 43, 387 (2013).

[9] M. Gharbi, Z. H. Sun, P. Sharma, and K. White, The origins of electromechanical indentation size effect in ferroelectrics, Appl. Phys. Lett. 95, 142901 (2009).

[10] C. R. Robinson, K. W. White, and P. Sharma, Elucidating the mechanism for indentation size-effect in dielectrics, Appl. Phys. Lett. 101, 122901 (2012).

[11] H. Zhou, Y. Pei, F. Li, H. Luo, and D. Fang, Electric-fieldtunable mechanical properties of relaxor ferroelectric single crystal measured by nanoindentation, Appl. Phys. Lett. 104, 061904 (2014).

[12] A. Abdollahi, C. Peco, D. Millán, M. Arroyo, G. Catalan, and I. Arias, Fracture toughening and toughness asymmetry induced by flexoelectricity, Phys. Rev. B 92, 094101 (2015).

[13] K. Cordero-Edwards, N. Domingo, A. Abdollahi, J. Sort, and G. Catalan, Ferroelectrics as smart mechanical materials, Adv. Mater. 29, 1702210 (2017).

[14] F. Vásquez-Sancho, A. Abdollahi, D. Damjanovic, and G. Catalan, Flexoelectricity in bones, Adv. Mater. 30, 1705316 (2018).

[15] A. Zukauskas, V. Pasiskevicius, and C. Canalias, Secondharmonic generation in periodically poled bulk $\mathrm{Rb}$-doped $\mathrm{KTiOPO}_{4}$ below $400 \mathrm{~nm}$ at high peak-intensities, Opt. Express 21, 1395 (2013).

[16] C. Liljestrand, A. Zukauskas, V. Pasiskevicius, and C. Canalias, Highly efficient mirrorless optical parametric oscillator pumped by nanosecond pulses, Opt. Lett. 42, 2435 (2017).

[17] A. C. Fischer-Cripps, Introduction to Contact Mechanics, 2nd ed. (Springer Press, New York, 2007).

[18] G. R. Anstis, P. Chantikil, B. R. Lawn, and D. B. Marshall, A critical evaluation of indentation techniques for measuring fracture toughness: I, direct crack measurements, J. Am. Ceram. Soc. 64, 533 (1979).

[19] H. Lu, C.-W. Bark, D. Esque de los Ojos, J. Alcalá, C. B. Eom, G. Catalán, and A. Gruverman, Mechanical writing of ferroelectric polarization, Science 336, 59 (2012).

[20] J. Ocenásek, H. Lu, C. W. Bark, C. B. Eom, J. Alcalá, G. Catalan, and A. Gruverman, Nanomechanics of flexoelectric switching, Phys. Rev. B 92, 035417 (2015).

[21] S. C. Hwang, C. S. Lynch, and R. M. McMeeking, Ferroelectric/ferroelastic interactions and a polarization switching model, Acta Metall. Mater. 43, 2073 (1995).

[22] X. Zeng and R. K. N.D. Rajapakse, Domain switching induced fracture toughness variation in ferroelectrics, Smart Mater. Struct. 10, 203 (2001). 
[23] G. A. Schneider, Influence of electric field and mechanical stresses on the fracture of ferroelectrics, Annu. Rev. Mater. Res. 37, 491 (2007).

[24] J. Narvaez, S. Saremi, J. Hong, M. Stengel, and G. Catalan, Large flexoelectric anisotropy in paraelectric barium titanate, Phys. Rev. Lett. 115, 037601 (2015).

[25] A. Zukauskas, G. Strömqvist, V. Pasiskevicius, F. Laurell, M. Fokine, and C. Canalias, Fabrication of submicrometer quasi-phase-matched devices in KTP and RKTP, Opt. Mater. Express 1, 1319 (2011).

[26] B. Lawn, Fracture of Brittle Solids, 2nd ed. (Cambridge University Press, Cambridge, England, 1993).

[27] W. R. Inc., Mathematica, Version 10. 2, (Wolfram Research, Inc., Champaign, IL, 2016).

[28] See Supplemental Material at http://link.aps.org/ supplemental/10.1103/PhysRevLett.122.135502 which includes the 2D maps of the strain field around a crack tip.

[29] R. T. Smith and F. S. Welsh, Temperature dependence of the elastic, piezoelectric, and dielectric constants of lithium tantalate and lithium niobate, J. Appl. Phys. 42, 2219 (1971).
[30] A. W. Warner, M. Onoe, and G. A. Coquin, Determination of elastic and piezoelectric constants for crystals in class (3m), J. Acoust. Soc. Am. 42, 1223 (1967).

[31] V. Gopalan, V. Dierolf, and D. A. Scrymgeour, Defectdomain wall interactions in trigonal ferroelectrics, Annu. Rev. Mater. Res. 37, 449 (2007).

[32] S. Haussuhls, S. Luping, W. Baolin, W. Jiyang, J. Liebertz, A. Wostrackc, and Ch. Fink, Physical properties of single crystals of $\mathrm{KTiPO}_{4}, \mathrm{~K}_{X} \mathrm{Rb}_{1-X} \mathrm{TiOPO}_{4}(X=0.85 ; 0.75), \mathrm{KGeOPO}_{4}$ and $\mathrm{KTiOAsO}_{4}$, Cryst. Res. Technol. 29, 583 (1994).

[33] J. D. Bierlein and H. Vanherzeele, Potassium titanyl phosphate: properties and new applications, J. Opt. Soc. Am. B 6, 622 (1989).

[34] J. Y. Jo, D. J. Kim, Y. S. Kim, S.-B. Choe, T. K. Song, J.-G. Yoon, and T. W. Noh, Polarization Switching Dynamics Governed by the Thermodynamic Nucleation Process in Ultrathin Ferroelectric Films, Phys. Rev. Lett. 97, 247602 (2006).

[35] J. Y. Jo, Y. S. Kim, T. W. Noh, J.-G. Yoon, and T. K. Song, Coercive fields in ultrathin $\mathrm{BaTiO}_{3}$ capacitors, Appl. Phys. Lett. 89, 232909 (2006). 